H. Umemura

Nagoya Math. J.

Vol. 157 (2000), 15-46

\title{
ON THE TRANSFORMATION GROUP OF THE SECOND PAINLEVÉ EQUATION
}

\author{
HIROSHI UMEMURA
}

\begin{abstract}
We show that for the second Painlevé equation $y^{\prime \prime}=2 y^{3}+t y+\alpha$, the Bäcklund transformation group $G$, which is isomorphic to the extended affine Weyl group of type $\hat{A}_{1}$, operates regularly on the natural projectification $\mathcal{X}(c) / \mathbb{C}(c, t)$ of the space of initial conditions, where $c=\alpha-1 / 2 . \mathcal{X}(c) / \mathbb{C}(c, t)$ has a natural model $\mathcal{X}[c] / \mathbb{C}(t)[c]$. The group $G$ does not operate, however, regularly on $\mathcal{X}[c] / \mathbb{C}(t)[c]$. To have a family of projective surfaces over $\mathbb{C}(t)[c]$ on which $G$ operates regularly, we have to blow up the model $\mathcal{X}[c]$ along the projective lines corresponding to the Riccati type solutions.
\end{abstract}

\section{$\S 1$. Introduction}

As is well known, the (extended) affine Weyl group of type $\tilde{A}_{1}$ appears as a transformation group of solutions of the second Painlevé equation

$$
P_{I I}(\alpha): y^{\prime \prime}=2 y^{3}+t y+\alpha,
$$

where $t$ is the independent variable, $y^{\prime \prime}=d^{2} y / d t^{2}$ and $\alpha \in \mathbb{C}$ is a parameter. If $y$ is a solution of the second Painlevé equation $P_{I I}(\alpha)$, then

$$
T_{+}(y)=-y-\frac{\alpha+\frac{1}{2}}{y^{\prime}+y^{2}+\frac{t}{2}}
$$

is a solution of $P_{I I}(\alpha+1)$,

$$
T_{-}(y)=-y+\frac{\alpha-\frac{1}{2}}{y^{\prime}-y^{2}-\frac{t}{2}}
$$

is a solution of $P_{I I}(\alpha-1)$ and $I(y)=-y$ is a solution of $P_{I I}(-\alpha)$. Let $G$ be the subgroup of the affine transformation group of the affine line $\mathbb{A}^{1}$ generated by the translations

$$
t_{+}(\alpha)=\alpha+1, \quad t_{-}(\alpha)=\alpha-1
$$

Received July 14, 1997. 
and the reflection

$$
i(\alpha)=-\alpha
$$

at 0 for $\alpha \in \mathbb{C}$. So $G$ is the affine Weyl group of type $\tilde{A}_{1}$. We consider the affine space $\mathbb{A}^{4}$ with coordinate system $\left(y, y^{\prime}, t, \alpha\right)$ as well as the affine plane with coordinate system $(t, \alpha)$. We have a vector field

$$
\delta(\alpha)=\frac{\partial}{\partial t}+y^{\prime} \frac{\partial}{\partial y}+\left(2 y^{3}+t y+\alpha\right) \frac{\partial}{\partial y^{\prime}}
$$

on $\mathbb{A}^{4}$ and a natural fibration $\pi: \mathbb{A}^{4} \rightarrow \mathbb{A}^{2}$ by projection $\left(y, y^{\prime}, t, \alpha\right) \mapsto(t, \alpha)$. The affine Weyl group $G$ operates on the affine plane $\mathbb{A}^{2}$ through the second coordinate. The transformations $T_{+}, T_{-}, I$ define a birational operation of the affine Weyl group $G$ on $\mathbb{A}^{4}$ compatible with the derivation $\delta(c)$ such that the fibration $\pi: \mathbb{A}^{4} \rightarrow \mathbb{A}^{2}$ is $G$-equivariant. We construct in this note a projective model of the fibration $\pi: \mathbb{A}^{4} \rightarrow \mathbb{A}^{2}$ on which the Weyl group $G$ operates regularly. In fact we construct a projective model $\mathcal{X}$ of the generic fiber of the fibration $\pi: \mathbb{A}^{4} \rightarrow \mathbb{A}^{2}$ such that the affine Weyl group $G$ operates regularly on $\mathcal{X}$ (Theorem 2.11). The model $\mathcal{X}$ is the projective surface studied by Okamoto [O1]. More precisely his space of initial conditions is our projective surface $\mathcal{X}$ minus 8 non-singular rational curves with self-intersection number -2 whose dual graph is the extended Dynkin diagram of type $\widetilde{E}_{7}$. We recall the construction of $\mathcal{X}$ in $\S 2$. We first projectify the affine plane $\mathbb{A}_{\mathbb{C}(\alpha, t)}^{2}$ to a ruled surface isomorphic to $\mathbb{P}^{1} \times \mathbb{P}^{1}$ and then blow up it 8 times to get $\mathcal{X}$. The construction of such a model $\mathcal{Y}$ over $\mathbb{A}^{2}$ is more subtle. We construct a model $\mathcal{Y}$ that is a complex manifold but is not an algebraic variety (Theorem 4.10). In this paper the ground field is an arbitrary field $K$ of characteristic 0 . So $K=\mathbb{Q}$ is the most natural but the readers who are interested in analysis may assume $K=\mathbb{C}$.

\section{$\S 2$. Construction of the model}

We know that the Painlevé equation

$$
P_{I I}(\alpha): y^{\prime \prime}=2 y^{3}+t y+\alpha
$$

is equivalent to both

$$
S_{I I}(\alpha):\left\{\begin{array}{l}
\frac{d q}{d t}=p-q^{2}-\frac{t}{2} \\
\frac{d p}{d t}=2 p q+\alpha+\frac{1}{2}
\end{array}\right.
$$


and

$$
S_{2}(c):\left\{\begin{array}{l}
\frac{d q}{d t}=q^{2}+p+\frac{t}{2}, \\
\frac{d p}{d t}=-2 q p+c
\end{array}\right.
$$

where $c=\alpha-1 / 2$ that is denoted by $\varepsilon$ by Okamoto (cf. [O1, p. 50]).

Remark. We consider that the parameter $\alpha$ or $c$ belongs to an extension field or more generally to an over ring of the base field $K$ consisting of constants.

We used $S_{I I}(\alpha)$ in [UW]. In this paper we adopt $S_{2}(\alpha)$. Let us denote by $\mathrm{Sol}_{2}(c)$ the set of solutions of the system $S_{2}(c)$. We have transformations

$$
\begin{gathered}
T_{+}(c, c+1): \operatorname{Sol}_{2}(c) \longrightarrow \operatorname{Sol}_{2}(c+1), \\
T_{-}(c, c-1): \operatorname{Sol}_{2}(c) \longrightarrow \operatorname{Sol}_{2}(c-1), \\
I(c,-c): \operatorname{Sol}_{2}(c) \longrightarrow \operatorname{Sol}_{2}(-c)
\end{gathered}
$$

The definition of these transformations is as follows. Let $(q, p) \in \operatorname{Sol}_{2}(c)$.

(i) If $2 q^{2}+q+1 \neq 0$, then

$$
T_{+}(c, c+1)(q, p)=\left(-q-\frac{c+1}{2 q^{2}+p+t},-2 q^{2}-p-t\right) .
$$

If $2 q^{2}+p+t=0$, then $c=-1$ and

$$
T_{+}(-1,0)(q, p)=\left(-q,-2 q^{2}-p-t\right)
$$

(ii) If $p \neq 0$, then

$$
T_{-}(c, c-1)(q, p)=\left(-q+\frac{c}{p},-p-2\left(q-\frac{c}{p}\right)^{2}-t\right) .
$$

If $p=0$, then $c=0$ and

$$
T_{-}(0,-1)(q, p)=\left(-q,-2 q^{2}-p-t\right)
$$

(iii) If $p \neq 0$,

$$
I(c,-c)(q, p)=\left(q-\frac{c}{p}, p\right)
$$


If $p=0$, then $c=0$ and $I(c,-c)(q, p)=(q, p)$. It is easy to check that

$$
\begin{gathered}
I(-c, c) \circ I(c,-c)=\operatorname{Id}_{S_{o_{2}}(c)}, \\
\left\{\begin{array}{l}
T_{-}(c+1, c) \circ T_{+}(c, c+1)=\operatorname{Id}_{S_{o l}(c)}, \\
T_{+}(c-1, c) \circ T_{-}(c, c-1)=\operatorname{Id}_{S o l_{2}(c)},
\end{array}\right.
\end{gathered}
$$

and

$$
I(-c+1, c-1) \circ T_{+}(-c,-c+1) \circ I(c,-c)=T_{-}(c, c-1)
$$

for every $c$. From now on, we assume that we assume that the parameter $c$ is a variable over $K(t)$ and it is convenient to set $L=K(c, t)$. Let now $q_{c}, p_{c}$ be variables over $L$ and we consider the polynomial ring $R(c):=L\left[q_{c}, p_{c}\right]$. If we consider the derivation

$$
D(c)=\frac{\partial}{\partial t}+\left(q_{c}^{2}+p_{c}+\frac{t}{2}\right) \frac{\partial}{\partial q_{c}}+\left(-2 q_{c} p_{c}+c\right) \frac{\partial}{\partial p_{c}}: R(c) \longrightarrow R(c),
$$

$R(c)$ is a differential algebra and we have

$$
\left\{\begin{array}{l}
D(c)\left(q_{c}\right)=q_{c}^{2}+q_{c}+\frac{t}{2}, \\
D(c)\left(p_{c}\right)=-2 q_{c} p_{c}+c,
\end{array}\right.
$$

i.e., $\left(q_{c}, p_{c}\right)$ is a solution of the system $S_{2}(c)$ and for every solution $(q, p)$ of $S_{2}(c)$ we have a differential $L$-morphism

$$
L\left[q_{c}, p_{c}\right] \longrightarrow L[q, p]
$$

of differential algebras sending $q_{a}, p_{a}$ respectively to $q, p$. In fact let $Q, P$ be differential variables over $L$ so that $L\{Q, P\}=L\left[Q, P, Q^{\prime}, P^{\prime}, \ldots\right]$ is a differential polynomial ring and we have a differential $L$-morphism

$$
\Phi: L\{Q, P\} \longrightarrow L[q, p]
$$

of differential $L$-algebras sending $Q, P$ respectively to $q, p$. Since the differential ideal $I(c)$ of the differential algebra $L\{q, p\}$ that is differentially generated by

$$
\delta Q-Q^{2}-P-\frac{t}{2}, \quad \text { and } \quad \delta P+2 Q P-c
$$


of the differential polynomial ring $L\{Q, P\}$ is in $\operatorname{Ker} \Phi$, the morphism $\Phi$ factors through the residue class map

$$
L\{Q, P\} \longrightarrow L\{Q, P\} / I(c)=R(c)
$$

and induces a differential $L$-morphism (2.6). We denote the quotient field of $R(c)$ by $Q(c)$, which is a differential field. We consider $X(c):=\operatorname{Spec} L\left[q_{c}, p_{c}\right]$ that is nothing but the affine plane $\mathbb{A}_{L}^{2}$ over $L$ with the coordinate system $\left(q_{c}, p_{c}\right)$ endowed with the derivation $\delta(c)$. Since $\left(q_{c}, p_{c}\right)$ is a solution of $S_{2}(c)$, it follows from $(2.1)$

$$
T_{+}(c, c+1)\left(q_{c}, p_{c}\right)=\left(-q_{c}-\frac{c+1}{2 q_{c}^{2}+p_{c}+t},-2 q_{c}^{2}-p_{c}-t\right)
$$

is a solution of $S_{2}(c+1)$. So by $(2.6)$ we have an $L$-differential morphism

$$
R(c+1) \longrightarrow Q(c)
$$

sending

$$
q_{c+1} \text { to }-q_{c}-\frac{c+1}{2 q_{c}^{2}+p_{c}+t} \text { and } p_{c+1} \text { to }-2 q_{c}^{2}-p_{c}-t
$$

Now by (2.4) the morphism (2.7) is birational or it induces an isomorphism

$$
Q(c+1) \longrightarrow Q(c)
$$

of the differential quotient fields. In other words we have an $L$-birational map

$$
X(c)=\mathbb{A}_{L}^{2}=\operatorname{Spec} R(c) \cdots \rightarrow X(c+1)=\mathbb{A}_{L}^{2}=\operatorname{Spec} R(c+1)
$$

compatible with the derivations $\delta(c+1)$ and $\delta(c)$. On the other hand the $K(t)$-isomorphism

$$
L=K(c, t) \longrightarrow L=K(c, t), \quad c \longmapsto c+1
$$

induces a differential $K(t)$-isomorphism

$$
R(c)=L\left[q_{c}, p_{c}\right] \longrightarrow R(c+1)=L\left[q_{c+1}, p_{c+1}\right]
$$

sending

$$
q_{c} \longmapsto q_{c+1}, \quad p_{c} \longmapsto p_{c+1} \quad \text { and } \quad c \longmapsto c+1
$$


Composing the isomorphism (2.9) and the $L$-birational map (2.8), we get a $K(t)$-birational map

$$
\begin{aligned}
T_{+}: X(c)=\operatorname{Spec} L\left[q_{c}, p_{c}\right] \longrightarrow X(c+1)=\operatorname{Spec} L\left[q_{c+1}, p_{c+1}\right] \\
\cdots \rightarrow X(c)=\operatorname{Spec} L\left[q_{c}, p_{c}\right]
\end{aligned}
$$

compatible with the derivations such that the diagram

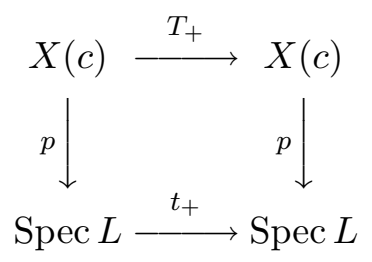

is commutative, where the vertical arrow $p$ is the projection and the lower horizontal arrow is the morphism of schemes induced by the isomorphism (2.9). Similarly we have a differential birational map

$$
T_{-}: X(c) \cdots \rightarrow X(c)
$$

such that the diagram

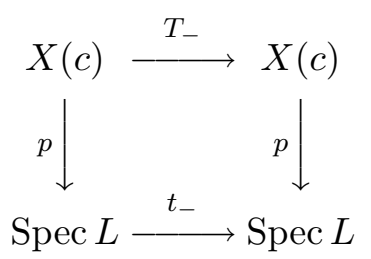

is commutative, where the lower horizontal arrow is the morphism of schemes induced by the $K(c)$-morphism $L \rightarrow L$ of differential fields sending $c$ to $c+1$. We also have a differential birational map

$$
I: X(c) \cdots \rightarrow X(c)
$$

such that the diagram

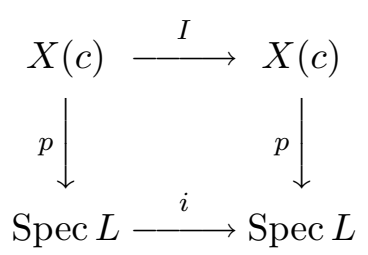


is commutative, where the lower horizontal map is induced by the $K(t)$ isomorphism $L \rightarrow L$ of differential fields sending $c$ to $-c$. Let

$$
t_{+}^{*}, \quad t_{-}^{*}, \quad i^{*}
$$

be respectively $K$-automorphisms of $K(c)$ such that

$$
t_{+}^{*}(c)=c+1, \quad t_{-}^{*}(c)=c-1, \quad i^{*}(c)=-c .
$$

They define $K$-automorphisms $t_{+}, t_{-}, i$ of the scheme $\operatorname{Spec} K(c)$. Let $G$ be the subgroup of the automorphism of the scheme Spec $K(t)$ generated by the automorphisms $t_{+}, t_{-}, i$ so that $G$ is the affine Weyl group of type $\tilde{A}_{1}$. We have

$$
t_{+}^{2}=t_{-}^{2}=t_{+} \circ t_{-}=t_{-} \circ t_{+}=i^{2}=\mathrm{Id}, \quad i \circ t_{+} \circ i=t_{-}
$$

and $G=\left\langle t_{+}, t_{-}\right\rangle \rtimes\langle i\rangle \simeq \mathbb{Z}^{2} \rtimes \mathfrak{S}_{2}$, where $\mathfrak{S}_{2}$ is the symmetric group of degree 2. Let $\widetilde{G}$ be the subgroup of the birational automorphisms of $X(c)$ generated by $T_{+}, T_{-}, I$. So we have a natural morphism $\varphi: \widetilde{G} \rightarrow G$ of groups by the commutative diagrams above. We can check

$$
T_{+} \circ T_{-}=T_{-} \circ T_{+}=S^{2}=\mathrm{Id}, \quad \text { and } \quad S \circ T_{+} \circ S=T_{-}
$$

so that $\varphi$ is an isomorphism. Namely the (extended) affine Weyl group $G$ of type $\tilde{A}_{1}$ birationally operates on the scheme $X(c)=\mathbb{A}_{L}^{2}$ in such a way that the diagram

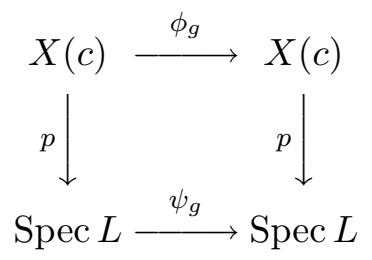

is commutative for every $g \in G$, where $\phi_{g}$ is the birational automorphism of $X(c)$ induced the element $g \in G$ and $\psi_{g}$ is the $K(t)$-automorphism of $\operatorname{Spec} L$ defined by the operation of the element $g \in G$. We projectify the affine plane $X(c)=\mathbb{A}_{L}^{2}$ with derivation. We prepare four copies $W_{i}(1 \leq i \leq 4)$ of the affine plane $\mathbb{A}_{L}^{2}$ and glue them by the following rule to get the projective model $Z(c)$ that is denoted by $\Sigma_{(\varepsilon)}^{(2)}$ in [O1] with $\varepsilon=c$. Let $\left(y_{i}, z_{i}\right)$ be the coordinate system of $W_{i}(1 \leq i \leq 4)$ so that $W_{i}=\operatorname{Spec} L\left[y_{i}, z_{i}\right]$. 
(i) A point $\left(y_{1}, z_{1}\right) \in W_{1}$ and a point $\left(y_{2}, z_{2}\right) \in W_{2}$ are identified if

$$
y_{1}=y_{2} \quad \text { and } \quad z_{1} z_{2}=1 .
$$

(ii) A point $\left(y_{1}, z_{1}\right) \in W_{1}$ and a point $\left(y_{3}, z_{3}\right) \in W_{3}$ are identified if

$$
y_{1} y_{3}=1 \quad \text { and } \quad z_{1}=c y_{3}-y_{3}^{2} z_{3} .
$$

We notice here that the latter condition is equivalent to $z_{3}=c y_{1}-y_{1}^{2} z_{1}$.

(iii) A point $\left(y_{3}, z_{3}\right) \in W_{3}$ and a point $\left(y_{4}, z_{4}\right) \in W_{4}$ are identified if

$$
y_{3}=y_{4} \quad \text { and } \quad z_{3} z_{4}=1 .
$$

The projections

$$
W_{i} \longrightarrow \mathbb{A}_{L}^{1}, \quad\left(y_{i}, z_{i}\right) \longmapsto y_{i}
$$

for $1 \leq i \leq 4$ glue together and give a morphism

$$
Z(c) \longrightarrow \mathbb{P}_{L}^{1}
$$

Namely $Z(c)$ is a $\mathbb{P}_{L}^{1}$-bundle over $\mathbb{P}_{L}^{1}$ or $Z(c)$ is a rational ruled surface known to be isomorphic to $\mathbb{P}^{1} \times \mathbb{P}^{1}$. The curves $z_{2}=0$ in $W_{2}$ and $z_{4}=0$ in $W_{4}$ also glue together and give a section $D_{1}$ of the ruled surface $Z(c) \rightarrow \mathbb{P}_{L}^{1}$ such that $D_{1}^{2}=2$. We embed the affine plane $X(c)$ over $L$ in $Z(c)$ by identifying it with $W_{1}$ by an $L$-isomorphism

$$
L\left[y_{1}, z_{1}\right] \longrightarrow L\left[q_{c}, p_{c}\right], \quad y_{1} \longmapsto q_{c}, z_{1} \longmapsto p_{c} .
$$

The derivation $\delta(c)$ of $L\left[q_{c}, p_{c}\right]$ defines a rational derivation on the scheme $Z(c)$. We notice here that since $\delta(t)=1$, the rational derivation on $Z(c)$ is not a vector field on an variety over $L$ even on an open set of $Z(c)$, for $Z(c)$ is defined over the field $L=K(t, c)$. Okamoto constructed the space of initial conditions of the second Painlevé equation $P_{I I}(\alpha)$ by blowing up the projective surface $Z(c)$ over $L$ at 8 points. They are infinitely near points of $\left(y_{4}, z_{4}\right)=(0,0)$ on $W_{4} \subset Z(c)$. Namely they are the point $\left(y_{4}, z_{4}\right)=(0,0)$ in $W_{4}$ and 7 other points lying on the exceptional divisor that is contracted to the point $\left(y_{4}, z_{4}\right)=(0,0) \in W_{4}$. Let us denote the thus obtained surface by $\mathcal{X}(c)$. On $\mathcal{X}(c)$ there are 8 curves $D_{i}$ isomorphic to $\mathbb{P}_{L}^{1}$ with $D_{i}^{2}=-2$ $(1 \leq i \leq 8)$. The space of initial conditions of the second Painleve equation is defined as $\mathcal{X}(c)-\bigcup_{i=1}^{8} D_{i}$ (cf. [O1, Chap. III, $\left.\S 1\right]$ ). 
TheOREM 2.11. The rational operation of the group $G$ on $X(c)$ gives a regular operation of $G$ on the projective model $\mathcal{X}(c)$ of $X(c)$.

We prove the Theorem in $\S 3$. To explain the construction of $\mathcal{X}(c)$, we need the notation. Let $W$ be the affine plane $A_{M}^{2}$ with a coordinate system $(y, z)$, i.e., $W=\operatorname{Spec} M[y, z]$, where $M$ is a field. The blow-up $p: \widetilde{W} \rightarrow W$ of $W$ at $(y, z)=(0,0)$ is by definition

$$
\widetilde{W}=\left\{\left(y, z ;\left(x_{0}, x_{1}\right)\right) \in W \times \mathbb{P}_{M}^{1} \mid y x_{0}=z x_{1}\right\}
$$

and the morphism $p: \widetilde{W} \rightarrow W$ is induced by the projection $W \times \mathbb{P}_{M}^{1} \rightarrow W$. Let us denote by $W(y)$ the open subset

$$
\left\{\left(y, z ;\left(x_{0}, x_{1}\right)\right) \in \widetilde{W} \mid x_{0} \neq 0\right\}
$$

of $\widetilde{W}$. Then writing $x_{1} / x_{0}=Y$, we have an isomorphism

$$
W(y) \longrightarrow \mathbb{A}_{M}^{2}, \quad\left(y, z ;\left(x_{0}, x_{1}\right)\right) \longmapsto\left(\frac{x_{1}}{x_{0}}, z\right)=(Y, z) .
$$

In fact the inverse map $\mathbb{A}_{M}^{2} \rightarrow W(y)$ is given by $(Y, z) \mapsto(Y z, z ;(1, Y))$. Namely the open subset $W(y) \in \widetilde{W}$ is isomorphic to the affine plane $\mathbb{A}_{M}^{2}$ with the coordinate system $(Y, z)$. Similarly we write $x_{0} / x_{1}=Z$. Then the open subset

$$
W(z):=\left\{\left(y, z ;\left(x_{0}, x_{1}\right)\right) \in \widetilde{W} \mid x_{1} \neq 0\right\}
$$

of $\widetilde{W}$ is isomorphic to $\mathbb{A}_{M}^{2}$ by sending $\left(y, z ;\left(x_{0}, x_{1}\right)\right)$ to $\left(y, x_{0} / x_{1}\right)=(y, Z)$. In other words the open subset $W(z) \subset \widetilde{W}$ is isomorphic to the affine plane $\mathbb{A}_{M}^{2}$ with the coordinate system $(y, Z)$. So $\widetilde{W}$ is covered by the two open subsets $W(y), W(z)$ isomorphic to the affine plane $\mathbb{A}_{M}^{2}$. On the open subset $W(y) \simeq \mathbb{A}_{L}^{2}$ of $\widetilde{W}$ the projection

$$
p: \widetilde{W} \longrightarrow W, \quad\left(y, z ;\left(x_{0}, x_{1}\right)\right) \longmapsto(y, z)
$$

is written in terms of the coordinate system $(Y, z)$ as

$$
(Y, z) \longmapsto(Y z, z)
$$

and similarly on the other open subset $W(z)$ of $\widetilde{W}$ the projection $p: \widetilde{W} \rightarrow$ $W$ is written in terms of the coordinate system $(y, Z)$ on $\mathbb{A}_{M}^{2}$ as

$$
(y, Z) \longmapsto(y, y Z) .
$$


Here is the construction of $\mathcal{X}(c)$. The center $a_{1}$ of the first blow-up of $Z(c)$ is the point $\left(y_{4}, z_{4}\right)=(0,0)$ on $W_{4}=\mathbb{A}^{2}$. Let $Z_{1}(c)$ be the blow-up of $Z(c)$ at $\left(y_{4}, z_{4}\right)=(0,0)$. Ignoring the index 4 , we use the above convention for $W_{4}$ and $M=L$. We have the blow-up

$$
p_{4}: \widetilde{W}_{4} \longrightarrow W_{4}
$$

and $\widetilde{W}_{4}$ is covered by two open subsets $W_{4}(y), W_{4}(z)$ both isomorphic to $\mathbb{A}_{L}^{2}$ with the coordinate systems $(Y, z)$ and $(y, Z)$. So $Z_{1}(c)$ is covered by 5 open subsets isomorphic to $\mathbb{A}^{2}: W_{1}, W_{2}, W_{3}, W_{4}(y), W_{4}(z)$. Then the center $a_{2}$ of the second blow-up of $Z_{1}(c)$ is the point $(y, Z)=(0,0)$ on $W_{4}(z)=\mathbb{A}^{2}$. We denote by $Z_{2}(c)$ the thus obtained surface. To simplify the notation, we set $W_{5}=W_{4}(z)$ that is the affine plane, and we denote the coordinate system $(y, Z)$ of the affine plane $W_{5}$ by $(y, z)$ which we should not confuse with the coordinate system on $W_{4}$. So we get the blow-up

$$
p_{5}: \widetilde{W}_{5} \longrightarrow W_{5}
$$

of $W_{5}$ at $(y, z)=(0,0) . \widetilde{W}_{5}$ is covered by two open subsets $W_{5}(y)$ and $W_{5}(z)$ with the coordinate systems $(Y, z)$ and $(y, Z)$ respectively both isomorphic to the affine plane $\mathbb{A}^{2}$. Therefore $Z_{2}(c)$ is covered by 6 affine planes:

$$
W_{1}, W_{2}, W_{3}, W_{4}(y), W_{5}(y), W_{5}(z) .
$$

This procedure is repeated to get the third and fourth centers $a_{3}, a_{4}$. Namely $W_{5}(z)$ is the affine plane with the coordinate system $(y, Z)$ and we set $W_{6}=W_{5}(z)$ and denote $Z$ by $z$ so that the coordinate system on the affine plane $W_{6}$ is $(y, z)$. The center $a_{3}$ of the third blow-up is the point $(y, z)=(0,0)$ of the affine plane $W_{6}=W_{5}(z) \subset Z_{2}(c)$. So we blow up $Z_{2}(c)$ at the point $(y, z)=(0,0)$ in $W_{6}$ to obtain $Z_{3}(c)$. Locally on $W_{6}$, we get the blow-up

$$
p_{6}: \widetilde{W}_{6} \longrightarrow W_{6}
$$

which is covered by two open subsets $W_{6}(y)$ and $W_{6}(z)$ that are affines planes with coordinate systems $(Y, z)$ and $(y, Z)$. So $Z_{3}(c)$ is covered by 7 affine planes

$$
W_{1}, W_{2}, W_{3}, W_{4}(y), W_{5}(y), W_{6}(y), W_{6}(z) .
$$

The center $a_{4}$ of the fourth blow-up to get $Z_{4}(c)$ is the point $(y, Z)=(0,0)$ on $W_{6}(z) \subset Z_{3}(c)$. So it is convenient to denote $Z$ by $z$ and we set $W_{7}=$ 
$W_{6}(z)$ so that $W_{7}$ is the affine plane with the coordinate system $(y, z)$. Locally we have the blow-up

$$
p_{7}: \widetilde{W}_{7} \longrightarrow W_{7}
$$

Then $\widetilde{W}_{7}$ is covered by two open subsets $W_{7}(y)$ and $W_{7}(z)$ isomorphic to the affine plane with the coordinate systems $(Y, z)$ and $(y, Z)$. So $Z_{4}(c)$ is covered by 8 affine planes:

$$
W_{1}, W_{2}, W_{3}, W_{4}(y), W_{5}(y), W_{6}(y), W_{7}(y), W_{7}(z)
$$

The center of the fifth blow-up is the point $(y, Z)=(0,1 / 2)$ of $W_{7}(z)$. So we denote $W_{7}(z)$ by $W_{8}$. Now we introduce a new coordinate system $(y, z)$ on the affine plane $W_{7}(z)$ by setting $y=y, Z=(z+1) / 2$. We use this new coordinate system on $W_{8}$ so that the center $a_{5}$ of the fifth blow-up is the point $(y, z)=(0,0)$ on $W_{8}$ in terms of the new coordinate system. Therefore we get the blow-up

$$
Z_{5}(c) \longrightarrow Z_{4}(c)
$$

Locally on $W_{8}$ we have the blow-up

$$
p_{8}: \widetilde{W}_{8} \longrightarrow W_{8}
$$

and $\widetilde{W}_{8}$ is covered by two open subsets $W_{8}(y)$ and $W_{8}(z)$ that have the coordinate systems $(Y, z)$ and $(y, Z)$ respectively. So $Z_{5}(c)$ is covered by 9 affine planes:

$$
W_{1}, W_{2}, W_{3}, W_{4}(y), W_{5}(y), W_{6}(y), W_{7}(y), W_{8}(y), W_{8}(z)
$$

The center $a_{6}$ of the sixth blow-up to get $Z_{6}(c)$ is the point $(y, Z)=(0,0)$ of $W_{8}(z)$. Therefore to simplify the notation, we set $W_{9}=W_{8}(z)$ and we denote $Z$ by $z$ so that $W_{9}$ is the affine plane with the coordinate system $(y, z)$. We get the blow-up

$$
Z_{6}(c) \longrightarrow Z_{5}(c)
$$

and locally we have a blow-up morphism

$$
p_{9}: \widetilde{W_{9}} \longrightarrow W_{9}
$$


So the surface $\widetilde{W}_{9}$ is covered by two open subsets $W_{9}(y)$ and $W_{9}(z)$ isomorphic to the affine plane with coordinate systems $(Y, z)$ and $(z, Y)$ respectively. The surface $Z(6)$ is covered by 10 affine planes:

$$
W_{1}, W_{2}, W_{3}, W_{4}(y), W_{5}(y), W_{6}(y), W_{7}(y), W_{8}(y), W_{9}(y), W_{9}(z) .
$$

The center of the seventh blowing up is $(y, Z)=(0,-t / 2)$ on $W_{9}(z) \subset Z_{6}(c)$. So we set $W_{10}=W_{9}(z)$ and introduce $z$ by $Z=(z-t) / 2$ and we use a new coordinate system $(y, z)$ on the affine plane $W_{10}$. So the center $a_{7}$ of the seventh blow-up is $(y, z)=(0,0)$ on $W_{10}$. In this way we get the blow-up

$$
Z_{7}(c) \longrightarrow Z_{6}(c)
$$

Locally we have the blow-up

$$
p_{10}: \widetilde{W}_{10} \longrightarrow W_{10}
$$

and the surface $\widetilde{W}_{10}$ is covered by open subsets $W_{10}(y)$ and $W_{10}(z)$ both isomorphic to the affine plane $\mathbb{A}^{2}$ with the coordinate systems $(Y, z)$ and $(y, Z)$. So $Z_{7}(c)$ is covered by 11 affine planes:

$W_{1}, W_{2}, W_{3}, W_{4}(y), W_{5}(y), W_{6}(y), W_{7}(y), W_{8}(y), W_{9}(y), W_{10}(y), W_{10}(z)$.

The center of the eighth and hence the last blow-up is the point $(y, Z)=$ $(0,-2 c-1)$ on $W_{10}(z)$. So we get

$$
Z_{8}(c) \longrightarrow Z_{7}(c)
$$

This the definition of $Z_{8}(c)=\mathcal{X}(c)$.

\section{$\S 3$. Proof of the Theorem}

The group $G$ is a Coxeter group generated by two reflections $i$ and $j$ :

$$
i: K(c) \longrightarrow K(c), \quad i(c)=-c, \quad \text { and } \quad j: K(c) \longrightarrow K(c), j(c)=-1-c .
$$

So we have to show that the birational automorphisms of $\mathcal{X}(c)$ corresponding to $i, j$ are in fact biregular automorphisms of $\mathcal{X}(c)$. Let us first study the reflection $j$. The operation of the reflection $j$ comes from the transformation

$$
\begin{gathered}
J(c,-1-c)=I(c+1,-1-c) \circ T_{+}(c, c+1): \operatorname{Sol}_{2}(c) \longrightarrow \operatorname{Sol}_{2}(-1-c), \\
(q, p) \longmapsto\left(-q,-2 q^{2}-p-t\right) .
\end{gathered}
$$


Keeping the notation of $\S 2$, we consider a differential $L$-morphism

$$
L\{Q, P\} \longrightarrow L\left(q_{c}, p_{c}\right)
$$

sending

$$
Q \longmapsto-q_{c}, \quad P \longmapsto-2 q_{c}^{2}-p_{c}-t .
$$

Since $J(c,-1-c)\left(q_{c}, p_{c}\right)=\left(-q_{c},-2 q_{c}^{2}-p_{c}-t\right) \in \operatorname{Sol}_{2}(-1-c)$, the morphism (3.1) factors through the residue class morphism

$$
L\{Q, P\} \longrightarrow L\{Q, P\} / I(-1-c)=R(-1-c)
$$

so that we have a differential $L$-morphism

$$
R(-1-c) \longrightarrow K(c)
$$

Since $J(-1-c, c) \circ J(c,-1-c)(q, p)=(q, p)$ for a generic solution $(q, p)$ of $S_{2}(c)$ over $L$, the $L$-morphism (3.2) is birational. Geometrically we have a differential $L$-birational map

$$
J_{X}(c,-1-c): X(c)=\operatorname{Spec} R(c) \cdots \rightarrow X(-1-c)=\operatorname{Spec} R(-1-c)
$$

and therefore differential $L$-birational maps

$$
\begin{aligned}
& J_{Z}(c,-1-c): Z(c) \cdots \rightarrow Z(-1-c), \text { and } \\
& J_{\mathcal{X}}(c,-1-c): \mathcal{X}(c) \cdots \rightarrow \mathcal{X}(-1-c),
\end{aligned}
$$

as $Z(c)$ and $\mathcal{X}(c)$ are models of $X(c)=$ Spec $R(c)$. Since we have a natural differential $K(t)$-isomorphism

$$
\begin{gathered}
R(c)=L\left[q_{c}, p_{c}\right] \longrightarrow R(-c-1)=L\left[q_{-1-c}, p_{-1-c}\right], \\
q_{c} \longmapsto q_{-1-c}, p_{c} \longmapsto p_{-1-c}, c \longmapsto-1-c,
\end{gathered}
$$

and thus a $K(t)$-isomorphism $X(-1-c)=\operatorname{Spec} R(-1-c) \rightarrow X(c)=$ Spec $R(c)$. This isomorphism gives further differential $K(t)$-isomorphisms

$$
J_{Z}^{\prime}: Z(-1-c) \longrightarrow Z(c) \text { and } \quad J_{\mathcal{X}}^{\prime}: \mathcal{X}(-c-1) \longrightarrow \mathcal{X}(c)
$$

Composing the morphisms (3.3), (3.4), we get birational maps

$$
J_{Z}: Z(c) \cdots \rightarrow Z(c) \text { and } \quad J_{\mathcal{X}}: \mathcal{X}(c) \cdots \rightarrow \mathcal{X}(c)
$$


that is by definition the birational operation of $j$ on $\mathcal{X}(c)$. We must show that $J=J_{\mathcal{X}}$ is an isomorphism. Since the morphisms (3.4) are isomorphisms, we have to show that the birational map $J_{\mathcal{X}}(c,-1-c): \mathcal{X}(c) \cdots \rightarrow$ $\mathcal{X}(-1-c)$ is biregular. To this end we look for the minimum resolution of the rational map

$$
J_{Z}(c,-1-c): Z(c) \cdots \rightarrow Z(-1-c)
$$

Indeed we see below that the blow-up $\mathcal{X}(c) \rightarrow Z(c)$ is the minimum resolution of the birational map $J_{Z}(c,-1-c): Z(c) \cdots \rightarrow Z(-1-c)$.

LEMmA 3.5. The blow-up $\mathcal{X}(c) \rightarrow Z(c)$ is the minimum resolution of the birational map $J_{Z}(c,-1-c): Z(c) \cdots \rightarrow Z(-1-c)$.

Let us admit for a moment Lemma 3.5. We have a regular map

$$
J_{\mathcal{X} Z}(c,-1-c): \mathcal{X}(c) \longrightarrow Z(-1-c)
$$

such that

$$
J_{Z}(c,-1-c) \circ p=J_{\mathcal{X} Z}(c,-1-c),
$$

$p: \mathcal{X}(c) \rightarrow Z(c)$ being the blow-up morphism. Since

$$
J(-1-c, c) \circ J(c,-1-c)=\operatorname{Id}_{\mathcal{X}(c)},
$$

the blow-up $\mathcal{X}(-1-c) \rightarrow Z(-1-c)$ is the minimum resolution of the birational map

$$
J_{Z}(c,-1-c)^{-1}=J_{Z}(-1-c, c): Z(-1-c) \cdots \rightarrow Z(c) .
$$

Therefore the birational map $J_{\mathcal{X}}(c,-1-c): \mathcal{X}(c) \rightarrow \mathcal{X}(-1-c)$ is biregular. So it remains to prove Lemma 3.5.

Proof of Lemma 3.5. To study the rational map $J_{Z}(c,-1-c): Z(c)$ $\cdots \rightarrow Z(-1-c)$, we simplify the notation. The ruled surfaces $Z(c)$ and $Z(-1-c)$ are defined by coverings $W_{i}(1 \leq i \leq 4)$. To distinguish the covering of $Z(c)$ from that of $Z(-1-c)$, we denote the covering of $Z(c)$ by $W_{i}, 1 \leq i \leq 4$, and the covering of $Z(-1-c)$ by $\bar{W}_{i}, 1 \leq i \leq 4$. So it follows from the definition that the $L$-morphism $J_{Z}(c,-1-c): Z(c) \cdots \rightarrow Z(-1-c)$ is defined by

$$
J_{11}: W_{1} \longrightarrow \bar{W}_{1}, \quad\left(y_{1}, z_{1}\right) \longmapsto\left(\bar{y}_{1}, \bar{z}_{1}\right)=\left(-y_{1},-2 y_{1}^{2}-z_{1}-t\right) .
$$


So $J_{Z}(c,-1-c)$ is regular on the open subset $W_{1}$ of $Z(c)$. As $y_{2}=y_{1}$, $z_{2}=z_{1}^{-1}$, on $W_{2}$ we have

$$
J_{21}: W_{2} \cdots \rightarrow \bar{W}_{1}, \quad\left(y_{2}, z_{2}\right) \longmapsto\left(\bar{y}_{1}, \bar{z}_{1}\right)=\left(-y_{2},-2 y_{2}^{2}-\frac{1}{z_{2}}-t\right) .
$$

So $J_{21}$ is not defined on $W_{2} \cap\left\{z_{2}=0\right\}$. On the other hand since $\bar{y}_{2}=\bar{y}_{1}$, $\bar{z}_{2}=\bar{z}_{1}^{-1}$, we have

$$
J_{22}: W_{2} \longrightarrow \bar{W}_{2}, \quad\left(y_{2}, z_{2}\right) \longmapsto\left(-y_{2}, \frac{z_{2}}{-2 y_{2} z_{2}-1-t z_{2}}\right) .
$$

So on $W_{2} \cap\left\{-2 y_{2}^{2} z_{2}-1-t z_{2}=0\right\}$ the map $J_{Z}(c,-1-c)$ is not defined and the set of base points of $J_{Z}(c,-1-c)$ on $W_{2}$ is

$$
\left\{2 y_{2}^{2}+1+t z_{2}=0\right\} \cap\left\{z_{2}=0\right\}=\emptyset .
$$

Namely $J_{Z}(c,-1-c)$ is regular on $W_{2}$. Similarly it follows from the definition of the ruled surface $Z(c)$ that we have on $W_{3}$

$$
\begin{aligned}
J_{31}: W_{3} \cdots \rightarrow \bar{W}_{1}, \quad\left(y_{3}, z_{3}\right) \longmapsto\left(-\frac{1}{y_{3}}, \frac{-2-\left(c y_{3}-y_{3}^{2} z_{3}\right) y_{3}^{3}-t y_{3}^{2}}{y_{3}^{2}}\right), \\
J_{32}: W_{3} \cdots \rightarrow \bar{W}_{2}, \quad\left(y_{3}, z_{3}\right) \longmapsto\left(-\frac{1}{y_{3}}, \frac{y_{3}^{2}}{-2-\left(c-y_{3} z_{3}\right) y_{3}^{3}-t y_{3}^{2}}\right), \\
J_{33}: W_{3} \cdots \rightarrow \bar{W}_{3}, \quad\left(y_{3}, z_{3}\right) \longmapsto\left(-y_{3}, \frac{2-z_{3} y_{3}^{4}+t y_{3}^{2}+(2 c+1) y_{3}^{2}}{y_{3}^{4}}\right)
\end{aligned}
$$

and

$$
J_{34}: W_{3} \cdots \rightarrow \bar{W}_{4}, \quad\left(y_{3}, z_{3}\right) \longmapsto\left(-y_{3}, \frac{y_{3}^{4}}{2-z_{3} y_{3}^{4}+t y_{3}^{2}+(2 c+1) y_{3}^{2}}\right) .
$$

So the rational map $J_{Z}(c,-1-c): Z(c) \cdots \rightarrow Z(-1-c)$ is regular on the open subset $W_{3}$ of $Z(c)$. Similarly an easy calculation shows

$$
\begin{aligned}
& J_{41}: W_{4} \cdots \rightarrow \bar{W}_{1}, \quad\left(y_{4}, z_{4}\right) \longmapsto\left(-\frac{1}{y_{4}}, \frac{-2 z_{4}-\left(c z_{4}-y_{4}\right) y_{4}^{3}-t y_{4}^{2} z_{4}}{y_{4}^{2} z_{4}}\right) \\
& J_{42}: W_{4} \cdots \rightarrow \bar{W}_{2}, \quad\left(y_{4}, z_{4}\right) \longmapsto\left(-\frac{1}{y_{4}}, \frac{y_{4}^{2} z_{4}}{-2 z_{4}-\left(c z_{4}-y_{4}\right) y_{4}^{3}-t y_{4}^{2} z_{4}}\right) \\
& J_{43}: W_{4} \cdots \rightarrow \bar{W}_{3}, \quad\left(y_{4}, z_{4}\right) \longmapsto\left(-y_{4}, \frac{2 z_{4}-y_{4}^{4}+t y_{4}^{2} z_{4}+(2 c+1) y_{4}^{3} z_{4}}{y_{4}^{4} z_{4}}\right)
\end{aligned}
$$


and

$$
J_{44}: W_{4} \cdots \rightarrow \bar{W}_{4}, \quad\left(y_{4}, z_{4}\right) \longmapsto\left(-y_{4}, \frac{y_{4}^{4} z_{4}}{2 z_{4}-y_{4}^{4}+t y_{4}^{2} z_{4}+(2 c+1) y_{4}^{3} z_{4}}\right) .
$$

We conclude now that on $W_{4}$ the rational map

$$
J_{Z}(c,-1-c): Z(c) \cdots \rightarrow Z(-1-c)
$$

has a base point at $\left(y_{4}, z_{4}\right) \in W_{4}$ for which

$$
y_{4}^{4} z_{4}=0 \quad \text { and } \quad 2 z_{4}-y_{4}^{4}+t y_{4}^{2} z_{4}+(2 c+1) y_{4}^{3} z_{4}=0 .
$$

Namely $\left(y_{4}, z_{4}\right)=(0,0) \in W_{4}$ is the unique base point of the rational map $J_{Z}(c,-1-c): Z(c) \cdots \rightarrow Z(-1-c)$. This point is the center $a_{1}$ of the first blow-up in the construction of $\mathcal{X}(c)$ as well as in the resolution of the rational map $J_{Z}(c,-1-c)$. We have proved

Sublemma 3.6. The resolution of the rational map

$$
J_{Z}(c,-1-c): Z(c) \cdots \rightarrow Z(-1-c)
$$

is equivalent to the resolution of the rational function

$$
F: W_{4} \cdots \rightarrow \mathbb{P}^{1}, \quad\left(y_{4}, z_{4}\right) \longmapsto\left(y_{4}^{4} z_{4}, 2 z_{4}-y_{4}^{4}+t y_{4}^{2} z_{4}+(2 c+1) y_{4}^{3} z_{4}\right)
$$

on $W_{4}$.

We use the notation of the construction of the model $\mathcal{X}(c)$. We blow up $W_{4}$ at $a_{1}=\left(y_{4}, z_{4}\right)=(0,0)$ to get

$$
p_{4}: \widetilde{W}_{4} \longrightarrow W_{4}
$$

$\widetilde{W}_{4}$ is covered by the two affine planes $W_{4}(y)$ and $W_{4}(z)$ with the coordinate systems respectively $(Y, z)$ and $(z, Y)$. In terms of these coordinate systems the morphism $p_{4}$ is written as

$$
W_{4}(y)=\mathbb{A}^{2} \longrightarrow W_{4}=\mathbb{A}^{2}, \quad(Y, z) \longmapsto\left(y_{4}, z_{4}\right)=(Y z, z)
$$

on $W_{4}(y)$ and

$$
W_{4}(z)=\mathbb{A}^{2} \longrightarrow W_{4}=\mathbb{A}^{2}, \quad(y, Z) \longmapsto\left(y_{4}, z_{4}\right)=(y, y Z)
$$


on $W_{4}(z)$. So we substituting (3.7) and (3.8), the rational function $F$ on $\widetilde{W}_{4}$ is given on $W_{4}(y)$ by

$$
\begin{gathered}
W_{4}(y)=\mathbb{A}^{2} \cdots \rightarrow \mathbb{P}^{1} \\
(Y, z) \longmapsto\left(Y^{4} z^{4}, 2-Y^{4} z^{3}+t Y^{2} z^{2}+(2 c+1) Y^{3} z^{3}\right)
\end{gathered}
$$

and on $W_{4}(z)$ by

$$
\begin{gathered}
W_{4}(z)=\mathbb{A}^{2} \cdots \rightarrow \mathbb{P}^{1}, \\
(y, Z) \longmapsto\left(y^{4} Z, 2 Z-y^{3}+t y^{2} Z+(2 c+1) y^{3} Z\right) .
\end{gathered}
$$

It follows from (3.10) that the unique base point of the rational function $F$ on $W_{4}(z)$ is $(y, Z)=(0,0)$ and by $(3.9)$ the point $(Y, z)=(0,0) \in W_{4}(y)$ is not a base point. Thus $(y, Z)=(0,0)$ on $W_{4}(z)$ is the unique base point of the rational function $F$ on $\widetilde{W}_{4}$. This point coincides with the center $a_{2}$ of the second blow-up in the construction of $\mathcal{X}(c)$. Now we set $W_{5}=W_{4}(z)$ and denote $Y$ again by $y$. We blow up $W_{5}=\mathbb{A}^{2}$ with coordinate system $(y, z)$ at $a_{2}=(y, z)=(0,0) \in W_{5}$ to solve the singularity of the rational map (3.10), which is given by

$$
\begin{gathered}
W_{4}(z)=\mathbb{A}^{2} \cdots \rightarrow \mathbb{P}^{1} \\
(y, z) \longmapsto\left(y^{4} z, 2 z-y^{3}+t y^{2} z+(2 c+1) y^{3} z\right)
\end{gathered}
$$

because we denote $Y$ by $y$. Let $p_{5}: \widetilde{W}_{5} \rightarrow W_{5}$ be the blow-up so that $\widetilde{W}_{5}$ is covered by the two affine planes $W_{5}(y)$ and $W_{5}(z)$ with the coordinate systems $(Y, z)$ and $(y, Z)$. The morphism

$$
p_{5}: \widetilde{W}_{5} \longrightarrow W_{5}
$$

is given on the open subsets of $\widetilde{W}_{5}$ by

$$
W_{5}(y)=\mathbb{A}^{2} \longrightarrow W_{5}=\mathbb{A}^{2}, \quad(Y, z) \longmapsto(Y z, z)
$$

on $W_{5}(y)$ and

$$
W_{5}(z)=\mathbb{A}^{2} \longrightarrow W_{5}=\mathbb{A}^{2}, \quad(y, Z) \longmapsto(y, y Z)
$$

on $W_{5}(z)$. So if we substitute $(y, z)=(Y z, z)$ and $(y, z)=(y, y Z)$ into (3.11), we get the expressions of the rational function $F$ :

$$
W_{5}(y)=\mathbb{A}^{2} \cdots \rightarrow \mathbb{P}^{1}, \quad(Y, z) \longmapsto\left(Y^{4} z^{4}, 2-Y^{2} z-t Y^{2} z^{2}+(2 c+1) Y^{3} z^{3}\right)
$$


on $W_{5}(y)$ and

$$
W_{5}(z)=\mathbb{A}^{2} \cdots \rightarrow \mathbb{P}^{1}, \quad(y, Z) \longmapsto\left(y^{4} Z, 2 Z-y^{2}+t y^{2} Z+(2 c+1) y^{3} Z\right)
$$

on $W_{5}(z)$. So $(y, Z)=(0,0) \in W_{5}(z)$ is the unique base point of the rational function $F$ on $\widetilde{W}_{5}$. This point coincides with the center $a_{3}$ of the third blowup in the construction of $\mathcal{X}(c)$. Now we use the notation of the construction of $\mathcal{X}(c)$. We denote $Z$ by $z$ so that $W_{6}=W_{5}(z)$ is the affine plane with coordinate system $(y, z)$. We blow up $W_{6}$ at $a_{3}=(y, z)=(0,0)$. Then the rational function $F$ on $W_{6}$ is written on the open subsets $W_{6}(y)$ and $W_{6}(z)$ as

$$
W_{6}(y)=\mathbb{A}^{2} \cdots \rightarrow \mathbb{P}^{1}, \quad(Y, z) \longmapsto\left(Y^{4} z^{4}, 2+O(1)\right),
$$

where $O(1)$ is an element of the ideal $(y, z)$ of $L[Y, z]$, and

$$
W_{6}(z)=\mathbb{A}^{2} \cdots \rightarrow \mathbb{P}^{1}, \quad\left(y^{4} Z, 2 Z-y+t y^{2} Z+(2 c+1) y^{3} Z\right) .
$$

So $(y, Z)=(0,0)$ is the unique base point of the rational function $F$ on $\widetilde{W}_{6}$. This point is the center $a_{4}$ of the fourth blow-up in the construction of $\mathcal{X}(c)$. Hence we denote $Z$ by $z$ so that $W_{7}=W_{6}(z)$ is the affine plane with coordinate system $(y, z)$. We blow up $W_{7}$ at $a_{4}=(y, z)=(0,0)$ to get $\widetilde{W}_{7} \rightarrow W_{7}$. Then on $\widetilde{W}_{7}$ the representations of the rational function $F$ are as follows.

$$
W_{7}(y) \cdots \rightarrow \mathbb{P}^{1}, \quad(Y, z) \longmapsto\left(Y^{4} z^{4}, 2+O(1)\right)
$$

and

$$
W_{7}(z) \cdots \rightarrow \mathbb{P}^{1}, \quad(y, Z) \longmapsto\left(y^{4} Z, 2 Z-y+t y^{2} Z+(2 c+1) y^{3} Z\right),
$$

where $O(1)$ is an element of the ideal $(Y, z)$ of $L[Y, z]$. Hence the unique base point of the rational function $F$ on $\widetilde{W}_{7}$ is $(y, Z)=(0,0)$ on $W_{7}(z)$ that is the center $a_{5}$ of the fifth blow-up in the construction of $\mathcal{X}(c)$. We denote $Z$ by $z$ so that $W_{7}(z)=W_{8}$ is the affine plane with coordinate system $(y, z)$. We blow up $W_{8}$ at $(y, z)=(0,0)$. We then have local expressions of the rational function $F$ on the open subsets $W_{7}(y)$ and $W_{7}(z)$ of $\widetilde{W}_{7}$. Namely

$$
W_{7}(y) \cdots \rightarrow \mathbb{P}^{1}, \quad(Y, z) \longmapsto\left(Y^{4} z^{4}, 2+O(1)\right)
$$

where $O(1)$ is an element of the ideal $(Y, z)$ of $L[Y, z]$, and

$$
W_{7}(z) \cdots \rightarrow \mathbb{P}^{1}, \quad(y, Z) \longmapsto\left(y^{4} Z, 2 Z-1+t y^{2} Z+(2 c+1) y^{3} Z\right) .
$$


Therefore the unique base point of the rational function $F$ on $\widetilde{W}_{8}$ is the point $(y, Z)=(0,1 / 2)$ on $W_{7}(z)$. So we blow up $W_{7}(z)$ at $a_{5}=(y, Z)=$ $(0,1 / 2)$. We introduced the coordinate system $(y, z)$ on $W_{7}(z)$ such that $Z=(z+1) / 2$ and denote the affine plane $W_{7}(z)$ with this coordinate system $(y, z)$ by $W_{8}$. So on $W_{8}=\mathbb{A}^{2}$ the rational function $F$ is expressed as

$$
\begin{gathered}
W_{8} \cdots \rightarrow \mathbb{P}^{1} \\
(y, z) \longmapsto\left(y^{4} z+y^{4}, 2 z+t y^{2}+(2 c+1) y^{3}+t y^{2} z+(2 c+1) y^{3} z\right) .
\end{gathered}
$$

On the open subsets $W_{9}(y)$ and $W_{9}(z)$ of the blow-up $\widetilde{W}_{8} \rightarrow W_{8}$, the rational function $F$ is written as

$$
W_{8}(y) \cdots \rightarrow \mathbb{P}^{1}, \quad\left(Y^{4} z^{4}+Y^{4} z^{3}, 2+O(1)\right)
$$

where $O(1)$ is an element of the ideal $(Y, z)$ of $L[Y, z]$, and

$$
\begin{gathered}
W_{8}(z) \cdots \rightarrow \mathbb{P}^{1}, \\
(y, Z) \longmapsto\left(y^{4} Z+y^{3}, 2 Z+t y+(2 c+1) y^{2}+t y^{2} z+(2 c+1) y^{3} z\right) .
\end{gathered}
$$

So the unique base point of the rational function $F$ on $\widetilde{W}_{8}$ is the point $(y, Z)=(0,0)$ in $W_{8}(z)$. This is the center $a_{6}$ of the sixth blow-up in the construction of $\mathcal{X}(c)$. So we denote $Z$ by $z$ and blow up the affine plane $W_{8}(z)=W_{9}$ with coordinate system $(y, z)$ at $a_{6}=(y, z)=(0,0)$. Then the local expression of the rational function $F$ on the open subsets $W_{9}(y)$ and $W_{9}(z)$ are as follows.

$$
W_{9}(y) \cdots \rightarrow \mathbb{P}^{1}, \quad(Y, z) \longmapsto\left(Y^{4} z^{4}+Y^{4} z^{3}, 2+O(1)\right),
$$

where $O(1)$ is an element of the ideal $(Y, z)$ of $L[Y, z]$, and

$$
\begin{gathered}
W_{9}(z) \cdots \rightarrow \mathbb{P}^{1}, \\
(y, Z) \longrightarrow\left(y^{4} Z+y^{2}, 2 Z+t+(2 c+1) y+t y^{2} z+(2 c+1) y^{3} z\right) .
\end{gathered}
$$

So the unique base point of the rational function $F$ on $\widetilde{W}_{9}$ is the point $(y, Z)=(0,-t / 2)$ on $W_{9}(z)$. This point is the center of the seventh blow-up in the construction of $\mathcal{X}(c)$. We introduced $z$ by $Z=(z-t) / 2$ and the affine plane $W_{9}(z)$ with the coordinate system $(y, z)$ was denoted by $W_{10}$. So the rational function $F$ on $W_{10}$ is given by

$$
\begin{gathered}
W_{10} \cdots \rightarrow \mathbb{P}^{1}, \\
(y, z) \longmapsto\left(y^{4}(z-t)+2 y^{2}, 2 z+2(2 c+1) y+(t+(2 c+1) y) y^{2}(z-t)\right) .
\end{gathered}
$$


We blow up $W_{10}$ at $a_{7}=(y, z)=(0,0)$ to get $\widetilde{W}_{10} \rightarrow W_{10}$. The local representation of the rational function $F$ on $\widetilde{W}_{10}$ are

$$
W_{10} \cdots \rightarrow \mathbb{P}^{1}, \quad(Y, z) \longmapsto\left(O(1), 2+O(1)^{\prime}\right)
$$

where $O(1), O(1)^{\prime}$ are elements of the ideal $(Y, z)$ of $L[Y, z]$, and

$$
\begin{gathered}
W_{10}(z) \cdots \rightarrow \mathbb{P}^{1} \\
(y, Z) \longmapsto\left(y^{3}(y Z-t)+2 y, 2 Z+2(2 c+1)+(t+(2 c+1) y) y(y Z-t)\right) .
\end{gathered}
$$

So the unique base point of the rational function $F$ on $\widetilde{W}_{10}$ is the point $(y, Z)=(0,-2 c-1)$ of $\widetilde{W}_{10}(z)$. This is the center of the eighth blow-up in the construction of $\mathcal{X}(c)$. We introduced $z$ by $Z=z-2 c-1$ and denoted the affine plane $W_{10}(z)$ with this new coordinate system $(y, z)$ by $W_{11}$. Then we blew up $W_{11}$ at $(y, z)=(0,0)$ to get $\widetilde{W}_{11} \rightarrow W_{11}$. The rational function $F$ on $W_{11}$ is written as

$$
\begin{gathered}
W_{11} \cdots \rightarrow \mathbb{P}^{1}, \\
(y, z) \longmapsto\left(y^{3}(y(z-2 c-1)-t)+2 y,\right. \\
2 z+(t+(2 c+1) y) y(y(z-2 c-1)-t)) .
\end{gathered}
$$

Now the local expressions of the rational function $F$ on $\widetilde{W}_{11}$ are

$$
W_{11}(y) \cdots \rightarrow \mathbb{P}^{1}, \quad(y, z) \longmapsto\left(O(1), 2+O(1)^{\prime}\right)
$$

and

$$
\begin{gathered}
W_{11}(z) \cdots \rightarrow \mathbb{P}^{1}, \\
(y, Z) \longmapsto\left(y^{2}(y(y Z-2 c-1)-t)+2,\right. \\
2 Z+(t+(2 c+1) y)(y(y Z-2 c-1)-t)),
\end{gathered}
$$

where $O(1), O(1)^{\prime}$ are elements of the ideal $(Y, z)$ of $L[Y, z]$. So the point $(Y, z)=(0,0)$ of $W_{11}(y)$ is not a base point of the rational function $F$. We show that there is no base point of $F$ on $W_{11}(z)$ either, i.e., $F$ has no base point on $\mathcal{X}(c)$. In fact let $(y, Z) \in W_{11}(z)$ be a base point of the rational function $F$. Then $y \neq 0$. Equating the coordinates of $\mathbb{P}^{1}$ in (3.12) equals to 0 , we have

$$
y^{2}(y(y Z-2 c-1)-t)+2=0
$$


and

$$
2 Z+(t+(2 c+1) y)(y(y Z-2 c-1)-t)=0 .
$$

It follows from (3.13)

$$
y(y Z-2 c-1)-t=-\frac{2}{y^{2}} .
$$

We substitute (3.15) into (3.14) to get

$$
2 Z+(t+(2 c+1) y)\left(-\frac{2}{y^{2}}\right)=0
$$

and hence

$$
-Z y^{2}+(2 c+1) y+t=0,
$$

which contradicts (3.15). So Lemma 3.5 is proved.

Now we have to study the operation of the reflection $i$. The operation of the reflection $i$ comes from the transformation $I(c,-c)$ in $\S 2$. In fact keeping the notation in $\S 2$, we consider a differential $L$-morphism

$$
L\{Q, P\} \longrightarrow L\left(q_{c}, p_{c}\right)
$$

sending

$$
Q \longmapsto q_{c}-\frac{c}{p_{c}}, \quad P \longmapsto p_{c}
$$

Since

$$
\left(q_{c}-\frac{c}{p_{c}}, p_{c}\right)
$$

is a solution of the system $S_{2}(c)$, the morphism (3.1) factors through the residue class morphism

$$
L(c, t)\{Q, P\} \longrightarrow L\{Q, P\} / I(-c)=R(-c)
$$

so that we have a differential $L$-morphism

$$
R(-c) \cdots \rightarrow K(c)
$$

Since $I \circ I(q, p)=(q, p)$ for a generic solution $(q, p)$ of $S_{2}(c)$ over $L$, the $L$-morphism (3.18) is birational. Namely we have a differential $L$-birational morphism

$$
X(c)=\operatorname{Spec} R(c) \cdots \rightarrow \operatorname{Spec} R(-c)=X(-c)
$$


as we have a natural $L$-isomorphism

$$
R(c)=L\left(q_{c}, p_{c}\right) \longrightarrow R(-c)=L\left(q_{-c}, p_{-c}\right)
$$

sending

$$
q_{c} \longmapsto q_{-c}, \quad p_{c} \longmapsto p_{-c}, \quad c \longmapsto-c
$$

and thus a natural $L$-morphism

$$
X(-c)=\operatorname{Spec} R(-c) \longrightarrow X(c)=\operatorname{Spec} R(c)
$$

Composing the morphisms (3.19) and (3.20), we get the birational map $I: X(c) \cdots \rightarrow X(c)$. It follows from the construction that the isomorphism (3.20) induces an isomorphism $Z(-c) \rightarrow Z(c)$. So we have to show that the birational morphism $I_{\mathcal{X}}(c,-c): \mathcal{X}(c) \rightarrow \mathcal{X}(-c)$ arising from (3.19) is biregular. The $L$-birational map (3.19) defines an $L$-birational map

$$
I_{Z}(c,-c): Z(c) \cdots \rightarrow Z(-c)
$$

of the projective surfaces. We denote the charts $W_{i} \simeq \mathbb{A}_{L}^{2}$ of $Z(c)$ by $W_{i}(c)$ and the coordinate system of the affine plane $W_{i}(c)=\mathbb{A}^{2}$ by $\left(y_{i}, z_{i}\right)$ and the coordinate system of the affine plane $W_{i}(-c)=\mathbb{A}^{2}$ by $\left(\bar{y}_{i}, \bar{z}_{i}\right)$ for $1 \leq i \leq 4$. So the rational map $I_{Z}(c,-c)$ is given by a rational map

$$
W_{1}(c)=\mathbb{A}^{2} \cdots \rightarrow W_{1}(-c)=\mathbb{A}^{2}, \quad\left(y_{1}, z_{1}\right) \longmapsto\left(\bar{y}_{1}, \bar{z}_{1}\right)=\left(y_{1}-\frac{c}{z_{1}}, z_{1}\right)
$$

in terms of the charts $W_{1}(c)$ and $W_{1}(-c)$. Since

$$
\bar{y}_{3}=\frac{1}{\bar{y}_{1}}, \quad \bar{z}_{3}=-c \bar{y}_{1}-\bar{y}_{1}^{2} \bar{z}_{1},
$$

it follows from (3.22) that the rational map (3.21) gives

$$
\begin{gathered}
W_{1}(c)=\mathbb{A}^{2} \cdots \rightarrow W_{3}(-c)=\mathbb{A}^{2} \\
\left(y_{1}, z_{1}\right) \longmapsto\left(\bar{y}_{3}, \bar{z}_{3}\right)=\left(\frac{z_{1}}{y_{1} z_{1}-c},-y_{1}\left(y_{1} z_{1}-c\right)\right) .
\end{gathered}
$$

So the rational map (3.21) has no base point on $W_{1}(c)$. Substituting $y_{1}=y_{2}$, $z_{1}=1 / z_{2}$, we conclude that the rational map (3.21) gives rational maps

$$
W_{2}(c) \cdots \rightarrow W_{1}(-c), \quad\left(y_{2}, z_{2}\right) \longmapsto\left(y_{2}-c z_{2}, \frac{1}{z_{2}}\right) .
$$


and

$$
W_{2}(c) \cdots \rightarrow W_{2}(-c), \quad\left(y_{2}, z_{2}\right) \longmapsto\left(y_{2}-c z_{2}, z_{2}\right) .
$$

So there is no base point of rational map (3.21) on $W_{2}(c)$ either. A similar calculation shows that the rational map (3.21) is written as a rational map

$$
W_{3}(c) \cdots \rightarrow W_{1}(-c), \quad\left(y_{3}, z_{3}\right) \longmapsto\left(\frac{z_{3}}{y_{3} z_{3}-1},\left(c-y_{3} z_{3}\right) y_{3}\right)
$$

and

$$
W_{3}(c) \cdots \rightarrow W_{3}(-c), \quad\left(y_{3}, z_{3}\right) \longmapsto\left(-\frac{c-y_{3} z_{3}}{z_{3}}, z_{3}\right) .
$$

Therefore there is no base point of the rational map (3.21) on $W_{3}(c)$. Similarly in terms of $W_{4}(c)$ and $W_{4}(-c)$, the rational map (3.21) gives an isomorphism

$$
W_{4}(c) \longrightarrow W_{4}(-c), \quad\left(y_{4}, z_{4}\right) \longmapsto\left(y_{4}-c z_{4}, z_{4}\right)
$$

so that there is no base point of the rational map (3.21) on $W_{4}(c)$ either. Namely the rational map (3.21) is indeed regular. Now since $I_{Z}(-c, c)$ o $I_{Z}(c,-c)=\mathrm{Id}$, the rational map $(3.21)$ is biregular. We have to show that the rational map (3.21) induces the biregular morphism $\mathcal{X}(c) \rightarrow \mathcal{X}(-c)$. Let $C_{0}(c)$ be the Zariski closure of the curve

$$
\left\{\left(y_{4}, z_{4}\right) \in W_{4} \mid 2 z_{4}-y_{4}^{4}+t y_{4}^{4} z_{4}+(2 c+1) y_{4}^{3} z_{4}=0\right\}
$$

in $Z_{0}(c), E_{i}(c) \subset Z_{i}(c)$ the exceptional curve of the $i$-th blow-up $Z_{i}(c) \rightarrow$ $Z_{i-1}(c)$, and $C_{i}(c)$ the proper transform of $C_{0}(c)$ by the blow-up

$$
Z_{i}(c) \longrightarrow Z_{i-1}(c) \longrightarrow \cdots \longrightarrow Z_{0}(c)=Z(c)
$$

for $1 \leq i \leq 8$. In the course of the proof of Lemma 3.5, we have proved

LEMMA 3.24. The center $a_{1}$ of the first blow-up is the point $\left(y_{4}, z_{4}\right)=$ $(0,0) \in W_{4}$ and the center $a_{i}$ of the $i$-th blow-up $Z_{i}(c) \rightarrow Z_{i-1}(c)$ is the intersection of the curve $C_{i-1}(c)$ and the exceptional divisor $E_{i-1}$ for $2 \leq$ $i \leq 8$. Namely we have $a_{i}=E_{i-1} \cap C_{i-1}(c)$ for $2 \leq i \leq 8$.

We denote the center $a_{i}$ on $Z_{i-1}(c)$ by $a_{i}(c)$ and the center $a_{i}$ on $Z_{i-1}(-c)$ by $a_{i}(-c)$ to distinguish them. Therefore we must show that at each step of blow-up the center $a_{i+1}(c)$ on $Z_{i}(c)$ is mapped to the center 
$a_{i+1}(-c)$ on $Z_{i}(-c)$. In fact (3.23) shows that the first center $a_{1}$ on $Z(c)$ is mapped to the first center on $Z(-c)$. To see the image of the successive centers, in view of Lemma 3.24 let us consider the image of the curve

$$
C_{0}(c) \cap W_{4}=\left\{2 z_{4}-y_{4}^{4}+t y_{4}^{2} z_{4}+(2 c+1) y_{4}^{3} z_{4}=0\right\} \subset W_{4} \subset Z(c) .
$$

Since $I_{Z}(c,-c)\left(y_{4}, z_{4}\right)=\left(y_{4}-c z_{4}, z_{4}\right)=\left(\bar{y}_{4}, \bar{z}_{4}\right)$, we substitute

$$
y_{4}=\bar{y}_{4}+c q \bar{z}_{4}, \quad z_{4}=\bar{z}_{4}
$$

to get

$$
\begin{aligned}
2 \bar{z}_{4}-\bar{y}_{4}^{4}-t \bar{y}_{4}^{2} \bar{z}_{4}+(-2 c+1) \bar{y}_{4}^{3} \bar{z}_{4}+\left(2 t+3 c \bar{y}_{4}\right) \bar{y}_{4} \bar{z}_{4}^{2} \\
+3\left(c t+(2 c+1) c \bar{y}_{4}\right) \bar{z}_{4}^{3}+(-2 c+1) c^{3} \bar{z}_{4}^{4}=0 .
\end{aligned}
$$

The Zariski closure of this curve is the image $\bar{C}$ of the curve $C_{0}(c)$. We show that the birational map $I_{i}: Z_{i}(c) \cdots \rightarrow Z_{i}(-c)$ induced by $(3.21)$ is a biregular isomorphism for $0 \leq i \leq 8$ by induction. More precisely we prove by induction on $j$ that $I_{j}: Z_{j}(c) \rightarrow Z_{j}(-c)$ is a biregular isomorphism and $I_{j}\left(a_{j+1}(c)\right)=a_{j}(-c)$ for $0 \leq j \leq 7$, which implies $I_{8}=I_{\mathcal{X}}(c,-c): \mathcal{X}(c)=$ $Z_{8}(c) \rightarrow \mathcal{X}(-c)=Z_{8}(-c)$ is a biregular isomorphism. In fact we have seen that the assertion holds for $i=0$. Hence we assume that for a number $i$ with $0 \leq i \leq 7$, the birational map $I_{j}: Z_{j}(c) \cdots \rightarrow Z_{j}(-c)$ is a biregular isomorphism and $I_{j}\left(a_{j+1}(c)\right)=a_{j+1}(-c)$ for $0 \leq j \leq i$. Then the birational map $I_{i+1}: Z_{i+1}(c) \cdots \rightarrow Z_{i+1}(-c)$ is a biregular isomorphism. We have to show that $I_{i+1}\left(a_{i+2}(c)\right)=a_{i+2}(-c)$. Since $a_{i+2}(c)=Z_{i+1}(c) \cap C_{i+1}(c)$ and since $I_{j}\left(E_{j}(c)\right)=E_{j}(-c)$ for $j \leq i+1$, we have to show that the proper transform $\bar{C}_{l}(c)$ of $\bar{C}_{0}(c)$ and the curve $C_{l}(-c)$, which is the proper transform of $C_{0}(-c)$, under the blow-up

$$
Z_{l}(-c) \longrightarrow Z_{i-1}(-c) \longrightarrow \cdots \longrightarrow Z_{0}(-c)=Z(-c)
$$

both intersect with $E_{l}(-c)$ at the same point for $1 \leq l \leq 7$. This follows from the defining equation (3.25) of the curve $\bar{C}_{0}(c)$ and from the defining equation

$$
2 \bar{z}_{4}-\bar{y}_{4}^{2}-t \bar{y}_{4}^{2} \bar{z}_{4}+(-2 c+1) \bar{y}_{4}^{3} \bar{z}_{4}=0
$$

of the curve $C(-c)$ on $W_{4}(-c)$. 


\section{$\S 4$. Equivariant fibration over the affine line}

We proved that the affine Weyl group of type $\tilde{A}_{1}$ operates regularly on the projective surface $\mathcal{X}(c)$ in such a way that the fibration $\mathcal{X}(c) \rightarrow$ $\operatorname{Spec} L=\operatorname{Spec} K(t)(c)$ is equivariant. It is, however, more natural to look for an equivariant model

$$
\mathcal{Y}[c] \longrightarrow \operatorname{Spec} K(t)[c]
$$

of

$$
\mathcal{X}(c) \longrightarrow \operatorname{Spec} L=\operatorname{Spec} K(t)(c)
$$

such that the affine Weyl group operates biregularly on $\mathcal{Y}[c]$. We constructed the projective surface $\mathcal{X}(c)$ over the field $K(t)(c)$. The construction works over the ring $K(t)[c]$ so that we get a model

$$
\mathcal{X}[c] \longrightarrow \operatorname{Spec} K(t)[c]
$$

that is a scheme with derivation. So the affine Weyl group operates on the scheme $\mathcal{X}[c]$ with derivation birationally such that the fibration

$$
\mathcal{X}[c] \longrightarrow \operatorname{Spec} K(t)[c]
$$

is equivariant. Namely first we construct $Z[c]$ over Spec $K(t)[c]=\mathbb{A}_{K(t)}^{1}$ by gluing 4 copies of $\mathbb{A}_{K(t)}^{2} \times_{K(t)}$ Spec $K(t)[c]=\mathbb{A}_{K(t)}^{3}$, which we denote by $W_{i} \times \mathbb{A}_{K(t)}^{1}$ or by $W_{i}[c], 1 \leq i \leq 4$, by the same rule as in the construction of the ruled surface $Z(c)$. Then we blow up $Z[c] 8$ times along the sections of $Z[c] \rightarrow \mathbb{A}_{K(t)}^{1}$. So $W_{i}[c]$ is the affine space $\mathbb{A}_{K(t)}^{3}$ with the coordinate system $\left(y_{i}, z_{i}, c\right), 1 \leq i \leq 4$. Similarly we have rational maps over $\operatorname{Spec} K(t)[c]$

$$
J_{\mathcal{X}}[c,-1-c]: \mathcal{X}[c] \cdots \rightarrow \mathcal{X}[-1-c]
$$

and

$$
I_{\mathcal{X}}[c,-c]: \mathcal{X}[c] \cdots \rightarrow \mathcal{X}[-c] .
$$

In other words, locally on $W_{1}[c], W_{1}[-c]$ and $W_{1}[-1-c], J_{\mathcal{X}}[c,-1-c]$, $I_{\mathcal{X}}[c,-c]$ are given respectively by

$$
\begin{gathered}
J_{\mathcal{X}}[c,-1-c]: W_{1}[c] \cdots \rightarrow W_{1}[-1-c], \\
\left(y_{1}, z_{1}, c\right) \longmapsto\left(-y_{1},-2 y_{1}^{2}-z_{1}-t,-1-c\right)
\end{gathered}
$$


and

$$
I_{\mathcal{X}}[c,-c]: W_{1}[c] \cdots \rightarrow W_{1}[-c], \quad\left(y_{1}, z_{1}, c\right) \longmapsto\left(y_{1}-\frac{c}{z_{1}}, z_{1},-c\right) .
$$

The argument of $\S 3$ allows us to prove the following

Lemma 4.1. $J_{\mathcal{X}}[c,-1-c]: \mathcal{X}[c] \cdots \rightarrow \mathcal{X}[-1-c]$ is a biregular isomorphism.

The curves

$$
\left\{\left(y_{1}, z_{1}, c\right) \in W_{1}[c] \mid z_{1}=c=0\right\}
$$

on $W_{1}[c]$ and

$$
\left\{\left(y_{3}, z_{3}, c\right) \in W_{3}[c] \mid z_{3}=c=0\right\}
$$

glue together to define a curve $F$ on $\mathcal{X}[c]$ isomorphic to $\mathbb{P}_{K(t)}^{1}$, for $z_{3}=$ $c y_{1}-y_{1}^{2} z_{1}$ on $W_{1}[c] \cap W_{3}[c]$. Unfortunately the rational map $I_{\mathcal{X}}[c,-c]$ : $\mathcal{X}[c] \cdots \rightarrow \mathcal{X}[-c]$ is not biregular.

Lemma 4.2. The base locus of the rational map $I_{\mathcal{X}}[c,-c]: \mathcal{X}[c] \cdots \rightarrow$ $\mathcal{X}[-c]$ is the curve $F$.

Proof. Locally on $W_{1}[c]$ and $W_{1}[-c]$, the rational map $I_{\mathcal{X}}[c,-c]$ is given by

$$
W_{1}[c] \cdots \rightarrow W_{1}[-c], \quad\left(y_{1}, z_{1}, c\right) \longmapsto\left(y_{1}-\frac{c}{z_{1}}, z_{1},-c\right)
$$

and on $W_{3}[c]$ and $W_{3}[-c]$

$$
W_{3}[c] \cdots \rightarrow W_{3}[-c], \quad\left(y_{3}, z_{3}, c\right) \longmapsto\left(y_{3}-\frac{c}{z_{3}}, z_{3},-c\right) .
$$

The argument of $\S 3$ shows that there is no base point outside $W_{1}[c] \cup W_{3}[c]$. So the rational map $I_{\mathcal{X}}[c,-c]$ is not regular only on the curve $F$.

Since $I_{\mathcal{X}}[c,-c]$ is not regular, we have to modify the model $\mathcal{X}[c] \rightarrow$ $\mathbb{A}_{K(t)}^{1}$. To this end we blow up $\mathcal{X}[c]$ along the curve $F$ to get $\mathcal{X}^{1}[c] \rightarrow \mathcal{X}[c]$.

LEMMA 4.4. The rational map

$$
I_{\mathcal{X}}[c,-c]: \mathcal{X}[c] \cdots \rightarrow \mathcal{X}[-c]
$$

induces a biregular isomorphism

$$
I_{\mathcal{X}}^{1}[c,-c]: \mathcal{X}^{1}[c] \longrightarrow \mathcal{X}^{1}[-c]
$$


Proof. Locally on $W_{1}[c]$, we blew up $W_{1}[c]$ along $z_{1}=c=0$. The blow-up of $W_{1}[c]$ along the curve $z_{1}=c=0$ is by definition

$$
\widetilde{W}_{1}[c]=\left\{\left(y_{1}, z_{1}, c ;\left(x_{0}, x_{1}\right)\right) \in W_{1}[c] \times \mathbb{P}_{K(t)}^{1} \mid z_{1} x_{0}=c x_{1}\right\}
$$

and the projection $p: \widetilde{W}_{1}[c] \rightarrow W_{1}[c]$ is the restriction to $\widetilde{W}_{1}[c]$ of the projection $p_{1}: W_{1}[c] \times \mathbb{P}_{K(t)}^{1} \rightarrow W_{1}[c]$ on to the first factor. Let us denote by $\widetilde{W}_{1}[c]\left(z_{1}\right)$ the open subset

$$
\left\{\left(y_{1}, z_{1}, c ;\left(x_{0}, x_{1}\right)\right) \in \widetilde{W}_{1}[c] \mid x_{0} \neq 0\right\}
$$

of $\widetilde{W}_{1}[c]$. Then setting $Z_{1}=x_{1} / x_{0}$, we have an isomorphism

$$
W_{1}[c]\left[z_{1}\right] \longrightarrow \mathbb{A}_{K(t)}^{3}, \quad\left(y_{1}, z_{1}, c ;\left(x_{0}, x_{1}\right)\right) \longmapsto\left(y, x_{1} / x_{0}, c\right)=\left(y_{1}, Z_{1}, c\right) .
$$

Namely $W_{1}[c]\left(z_{1}\right)$ is the affine space with the coordinate system $\left(y_{1}, Z_{1}, c\right)$. Similarly if we denote $x_{0} / x_{1}$ by $C$, then the open subset

$$
W[c](c):=\left\{\left(y_{1}, z_{1}, c ;\left(x_{0}, x_{1}\right)\right) \in \widetilde{W}_{1}[c] \mid x_{1} \neq 0\right\}
$$

is isomorphic to $\mathbb{A}_{K(t)}^{3}$ by sending $\left(y_{1}, z_{1}, c ;\left(x_{0}, x_{1}\right)\right)$ to $\left(y_{1}, z_{1}, x_{0} / x_{1}\right)$. So $W_{1}[c](c)$ is isomorphic to the affine space $\mathbb{A}_{K(t)}^{3}$ with the coordinate system $\left(y_{1}, z_{1}, C\right)$. So the blow-up $\widetilde{W}_{1}[c]$ is covered by two open subsets $W_{1}[c]\left(z_{1}\right)$ and $W_{1}[c](c)$ isomorphic to $\mathbb{A}_{K(t)}^{3}$. On each open subsets the projection $p$ is given by

$$
W_{1}[c](z) \longrightarrow W_{1}[c], \quad(y, Z, c) \longmapsto(y, c Z, c)
$$

on $W_{1}[c](z)$ and

$$
W_{1}[c](c) \longrightarrow W_{1}[c], \quad(y, z, C) \longmapsto(y, z, z C)
$$

on $W_{1}[c](c)$. Let us check that the rational map $\mathcal{X}^{1}[c] \cdots \rightarrow \mathcal{X}^{1}[-c]$ is regular on $W_{1}[c](z)$. Locally we have an expression

$$
\begin{gathered}
W_{1}[c](z) \longrightarrow W_{1}[c] \cdots \rightarrow W_{1}[-c], \\
(y, Z, c) \longmapsto(y, Z c, c) \longmapsto\left(y-\frac{1}{Z}, Z c,-c\right)
\end{gathered}
$$

so that the rational map $W_{1}[c](z) \cdots \rightarrow W_{1}[-c](z)$ is given by

$$
(y, Z, c) \longmapsto\left(y-\frac{1}{Z},-Z,-c\right)
$$


and $W_{1}[c](z) \cdots \rightarrow W_{3}[-c]$ by

$$
\begin{gathered}
(y, Z, c) \longmapsto\left(\frac{1}{y Z-1},-c\left(y-\frac{1}{Z}\right)-\left(y-\frac{1}{Z}\right)^{2} Z c,-c\right) \\
=\left(\frac{1}{y Z-1}, c y(1-y Z),-c\right) .
\end{gathered}
$$

This shows that the rational map

$$
I_{\mathcal{X}}^{1}[c,-c]: \mathcal{X}^{1}[c] \cdots \rightarrow \mathcal{X}^{1}[-c]
$$

is regular on $W_{1}[c](z)$. On the other hand the composite rational map

$$
W_{1}[c](c) \longrightarrow W_{1}[c] \cdots \rightarrow W_{1}[-c]
$$

is given by

$$
(y, z, C) \longmapsto(y, z, z C) \longmapsto(y-C, z,-z C)
$$

and hence $W_{1}[c](c) \cdots \rightarrow W_{1}[-c](-c)$ is given by

$$
(y, z, C) \longrightarrow(y-C, z,-z C) \text {. }
$$

So the rational map

$$
I_{\mathcal{X}}^{1}[c,-c]: \mathcal{X}^{1}[c] \cdots \rightarrow \mathcal{X}^{1}[-c]
$$

is regular on $W_{1}[c](C)$ and consequently on $\widetilde{W}_{1}[c]$. We have a local expression of

$$
I_{\mathcal{X}}^{1}: \mathcal{X}^{1}[c] \cdots \rightarrow \mathcal{X}^{1}[c]
$$

in terms of $W_{3}[c]$ and $W_{3}[-c]$ :

$$
W_{3}[c] \cdots \rightarrow W_{3}[-c], \quad\left(y_{3}, z_{3}, c\right) \longmapsto\left(-\frac{c-y_{3} z_{3}}{z_{3}}, z_{3}, c\right)=\left(y_{3}-\frac{c}{z_{3}}, z_{3}, c\right)
$$

as we have seen in $\S 3$. So the above argument shows that the rational map $I_{\mathcal{X}}^{1}[c,-c]: \mathcal{X}^{1}[c] \cdots \rightarrow \mathcal{X}^{1}[-c]$ is regular locally on the blow-up of $W_{3}[c]$ too. If we notice here that in the construction of $\mathcal{X}^{1}[c]$ the centers $a_{i}[c]$ are on $W_{4}[c]$ and hence outside of the $W_{1}[c] \cup W_{3}[c]$ and that $I_{\mathcal{X}}[c,-c]$ induces an isomorphism $W_{4}[c] \rightarrow W_{4}[-c]$ mapping the centers $a_{i}[c]$ to the centers $a_{i}[-c]$, the rational map

$$
I_{\mathcal{X}}^{1}[c,-c]: \mathcal{X}^{1}[c] \cdots \rightarrow \mathcal{X}^{1}[-c]
$$

is regular. Since

$$
I_{\mathcal{X}}^{1}[-c, c] \circ I_{\mathcal{X}}^{1}[c,-c]=\mathrm{Id}
$$

$I_{\mathcal{X}}^{1}[c,-c]$ is biregular. 
Now

$$
I_{\mathcal{X}}^{1}[c,-c]: \mathcal{X}^{1}[c] \longrightarrow \mathcal{X}^{1}[-c]
$$

is biregular but the birational map

$$
J_{\mathcal{X}}^{1}[c,-1-c]: \mathcal{X}^{1}[c] \cdots \rightarrow \mathcal{X}^{1}[-1-c]
$$

corresponding to $J_{\mathcal{X}}$ is not regular. To remedy this, we have to blow up $\mathcal{X}^{1}[c]$ at infinitely many curves that are mutually disjoint. So the resulting object is not a scheme any more but a pro-scheme, i.e., the projective limit of schemes. In our case what we are going to get is a complex manifold if $K=$ $\mathbb{C}$. Similarly as we obtained $\mathcal{X}[c]$, we can construct the ruled surface $Z(c)$ and its blow-ups $Z_{i}(c), 1 \leq i \leq 8$ over $\mathbb{A}_{K(t)}^{1}$. We denote the corresponding varieties by $Z[c]$ and $Z_{i}[c]$, the exceptional divisors on $Z_{i}[c]$ by $E_{i}[c]$ for $1 \leq i \leq 8$ so that $\mathcal{X}[c]=Z_{8}[c]$. The proper transform of $E_{i}[c]$ on $\mathcal{X}[c]=Z_{8}[c]$ is denoted by $D_{i+1}[c]$ for $1 \leq i \leq 8$. The proper transform by the blow-up morphism $p: \mathcal{X}[c] \rightarrow Z[c]$ of the divisor

$$
\overline{\left\{z_{2}=0 \text { on } W_{2}[c]\right\}}=\overline{\left\{z_{4}=0 \text { on } W_{4}[c]\right\}}
$$

on $Z[c]$ is denoted by $D_{1}[c]$. For $\bar{c} \in \bar{K}$, we denote by $\mathcal{X}[\bar{c}]$ the reduction of $\mathcal{X}[c] / K(t)[c]$ at $c=\bar{c}$, i.e., $\mathcal{X}[\bar{c}]$ is the fiber $\mathcal{X}[c]_{\bar{c}}$ over the rational point $c=\bar{c}$ of $\mathbb{A}_{K(t)}^{1}=\operatorname{Spec} K(t)[c] \cdot \mathcal{X}[\bar{c}]$ is a projective surface over $K(t)[\bar{c}]=K(t)$ with the derivation $D(\bar{c})$ that is written as

$$
D(\bar{c})=\frac{\partial}{\partial t}+\left(y_{1}^{2}+z_{1}+\frac{t}{2}\right) \frac{\partial}{\partial y_{1}}+\left(-2 y_{1} z_{1}+c\right) \frac{\partial}{\partial z_{1}}
$$

on the open subset $W_{1}(\bar{c})$. The following result is due to Okamoto ([O 1 , Chap. III, §1]).

Proposition 4.5. For every $\bar{c} \in \bar{K}$ regarded as a $\bar{K}$-rational point of $\mathbb{A}_{K(t)}^{1}$ so that we can speak of the reduction $\mathcal{X}[\bar{c}]$, the Zariski open subset $\mathcal{X}[\bar{c}]-\bigcup_{i=1}^{8} D_{i}[c]$ is the set of points $P$ of $\mathcal{X}[c]$ where the rational vector field $D[\bar{c}]$ is regular at $P$. Namely we have

$$
\mathcal{X}[\bar{c}]-\bigcup_{i=1}^{8} D_{i}[\bar{c}]=\left\{P \in \mathcal{X}[\bar{c}] \mid D(\bar{c}) \mathcal{O}_{P} \subset \mathcal{O}_{P}\right\} .
$$


Corollary 4.6. Let $\bar{c}_{1}, \bar{c}_{2} \in \bar{K}$ and $f: \mathcal{X}\left[\bar{c}_{1}\right] \rightarrow \mathcal{X}\left[\bar{c}_{2}\right]$ be an isomorphism of schemes with derivation. Then

$$
f\left(\bigcup_{i=1}^{8} D_{i}\left[\bar{c}_{1}\right]\right)=\bigcup_{i=1}^{8} D_{i}\left[\bar{c}_{2}\right]
$$

and $f$ induces an isomorphism

$$
\left(\mathcal{X}\left[\bar{c}_{1}\right]-\bigcup_{i=1}^{8} D_{i}\left[\bar{c}_{1}\right]\right) \longrightarrow\left(\mathcal{X}\left[\bar{c}_{2}\right]-\bigcup_{i=1}^{8} D_{i}\left[\bar{c}_{2}\right]\right) .
$$

Proof. This is a direct consequence of the Proposition.

Lemma 4.7. For $\bar{c} \in \bar{K}$, the reduction

$$
J_{\mathcal{X}}(\bar{c},-1-\bar{c}): \mathcal{X}[\bar{c}] \longrightarrow \mathcal{X}[-1-\bar{c}]
$$

of the birational map $J_{\mathcal{X}}[c,-1-c]$ is an isomorphism of schemes with derivation.

Proof. This is a consequence of Lemma 4.1.

Lemma 4.8. For $\bar{c} \in \bar{K}$, the reduction

$$
I_{\mathcal{X}}(\bar{c},-\bar{c}): \mathcal{X}[\bar{c}] \longrightarrow[-\bar{c}]
$$

of the birational map $I_{\mathcal{X}}[c,-c]$ is an isomorphism of schemes with derivation.

Proof. The birational map $I[c,-c]$ has the base locus $F$ lying over the point $c=0$ but the reduction $I_{\mathcal{X}}[0,0]$ is equivalent to the identity. Now the lemma follows from Lemma 4.2.

Lemma 4.9. For every integer $n$, the open subset

$$
\mathcal{X}^{0}[n]:=\mathcal{X}[n]-\bigcup_{i=1}^{8} D_{i}[n]
$$

contains a curve $F[n]$ that is isomorphic to $\mathbb{P}_{K(t)}^{1}$, tangent to the vector field $D(n)$. Moreover the curve $F[n]$ is the unique complete curve on $\mathcal{X}^{0}[n]$ tangent to the vector field $D[n]$. 
Proof. We know that the assertion of the corollary holds for $n=0$ (see $[\mathrm{UW}]$ ). Now the assertion follows from Corollary 4.6, Lemmas 4.7 and 4.8 .

Now we blow up $\mathcal{X}[c]$ at the infinitely many curves $F[n], n \in \mathbb{Z}$ to get $\mathcal{Y}[c] \rightarrow \mathcal{X}[c]$.

THEOREM 4.10. The affine Weyl group of type $\tilde{A}_{1}$ regularly operates on $\mathcal{Y}[c]$ such that the fibration $\mathcal{Y}[c] \rightarrow$ Spec $K(t)[c]$ is equivariant.

Proof. It follows from Lemmas 4.1 and 4.4 that $I_{\mathcal{X}}(c,-c)$ and $J_{X}(c,-1-c)$ induce biregular morphisms

$$
I_{\mathcal{Y}}[c,-c]: \mathcal{Y}[c] \longrightarrow \mathcal{Y}[-c] \text { and } \quad J_{\mathcal{Y}}[c,-1-c]: \mathcal{Y}[c] \longrightarrow \mathcal{Y}[-1-c] .
$$

For two variables $c, c^{\prime}$ over $K(t)$, we have a natural isomorphism $\mathcal{Y}[c] \rightarrow$ $\mathcal{Y}\left[c^{\prime}\right]$ covering the isomorphism

$$
f^{*}: \operatorname{Spec} K(t)[c] \longrightarrow \operatorname{Spec} K(t)\left[c^{\prime}\right]
$$

where $f: K(t)\left[c^{\prime}\right] \rightarrow K(t)[c]$ is the $K(t)$-isomorphism sending $c^{\prime}$ to $c$. So combining morphisms of this type with $I_{\mathcal{Y}}[c,-c]$ and $Y_{\mathcal{Y}}[c,-1-c]$, we get the morphisms

$$
I_{\mathcal{Y}}: \mathcal{Y}[c] \longrightarrow \mathcal{Y}[-c] \text { and } \quad J_{\mathcal{Y}}: \mathcal{Y}[c] \longrightarrow \mathcal{Y}[-1-c]
$$

covering respectively $i^{*}$ and $j^{*}$, where $i, j$ are automorphisms of $K(t)[c]$ such that $i(c)=-c, j(c)=-1-c$. $I_{\mathcal{Y}}$ and $J_{\mathcal{Y}}$ are operations of $i$ and $j$. So the theorem is proved.

What is the fiber $\mathcal{Y}[0]$ over $c=0$ of the fibration $\mathcal{Y}[c] \rightarrow \mathbb{A}_{K(t)}^{1}$ ? $\mathcal{Y}[0]$ has two irreducible components $\mathcal{X}[0]$ and the exceptional divisor contracted to the curve $F$. We describe the differential equation on the exceptional divisor. Let us work over $W_{1}[c](c)$ that is the affine space with the coordinate system $\left(y_{1}, z_{1}, C\right)$, where $c=z_{1} C$. So on $W_{1}[c](c)$ we have,

$$
\left\{\begin{array}{l}
\frac{d y_{1}}{d t}=y_{1}^{2}+z_{1}+\frac{t}{2} \\
\frac{d z_{1}}{d t}=-2 y_{1} z_{1}+C z_{1}, \\
\frac{d C}{d t}=-2 y_{1} C+C
\end{array}\right.
$$


The fibration $W_{1}[c](c) \rightarrow \mathbb{A}^{1}$ is given by $\left(y_{1}, z_{1}, C\right) \mapsto c=z_{1} C$. So

$$
\begin{aligned}
& \mathcal{Y}[0] \cap W_{1}[c](c) \\
& \quad=\left\{\left(y_{1}, z_{1}, C\right) \in W_{1}[c](c) \mid C=0\right\} \cup\left\{\left(y_{1}, z_{1}, C\right) \in W_{1}[c](c) \mid z_{1}=0\right\} .
\end{aligned}
$$

Here

$$
\mathcal{X}[0] \cap W_{1}[c] \simeq\left\{\left(y_{1}, z_{1}, C\right) \in W_{1}[c](c) \mid C=0\right\}
$$

and

(the exceptional fibre) $\cap W_{1}[c](c)=\left\{\left(y_{1}, z_{1}, C\right) \in W_{1}[c](c) \mid z_{1}=0\right\}$

Therefore on

$$
\text { (the exceptional fibre) } \cap W_{1}[c](c)
$$

that is the affine plane with the coordinate system $\left(y_{1}, C\right)$ the differential equation (4.11) reduces to the system

$$
\left\{\begin{array}{l}
\frac{d y_{1}}{d t}=y_{1}^{2}+\frac{t}{2} \\
\frac{d C}{d t}=-2 y_{1} C+C
\end{array}\right.
$$

So the differential equation on the exceptional divisor is of little interest.

\section{REFERENCES}

[MMT] T. Matano, A. Matsumiya and K. Takano, On some Hamiltonian structures on Painlevé systems II, to appear, J. Math. Soc. Japan.

[Mu Y. Murata, Rational solutions of the second and the fourth Painlevé equations, Funkcial. Ekvac., 28 (1985), 1-32.

[O1] K. Okamoto, Sur les feuilletages associeés aux équation du second ordre à points critiques fixes de P. Painlevé, Jap. J. Math., 5 (1979), 1-79.

[O2] Studies on the Painlevé equations, Math. Ann., 275 (1986), 221-255.

[ST] T. Shioda and K. Takano, On some Hamiltonian structures of Painlevé systems I, Funkcial. Ekvac., 40 (1997), 271-291.

[US] H. Umemura and M. Saito, Painlevé equations and deformations of rational surfaces with rational double points, preprint.

[UW] H. Umemura, and H. Watanabe, Solutions of the second and fourth Painlevé equations, Nagoya Math. J., 148 (1997), 151-198.

Graduate School of Mathematics

Nagoya University

Nagoya, 464-8602

Japan

umemura@math.nagoya-u.ac.jp 